\title{
COVID-19 OUTBREAK IN ITALY. REPORT OF 20 CANCER PATIENTS WITH COVID-19 TREATED AT HOME DURING THE FIRST WAVE
}

\author{
L. Cavanna ${ }^{1}$, S. Casali ${ }^{2}$, C. Citterio ${ }^{1}$, G. Gianlupi ${ }^{3}$, L. Pilla ${ }^{4}$ \\ ${ }^{1}$ Department of Oncology and Hematology, Oncology Unit, Piacenza General Hospital, Piacenza, Italy \\ ${ }^{2}$ Family doctor, Castell'Arquato, Piacenza, Italy \\ ${ }^{3}$ Family doctor, Bardi, Parma, Italy \\ ${ }^{4}$ Family doctor, Ponte dell'Olio, Piacenza, Italy
}

\section{CORRESPONDING AUTHOR:}

\section{Luigi Cavanna}

Department of Oncology and Hematology

Oncology Unit

Piacenza General Hospital

via Taverna 49

2912 Piacenza, Italy

E-mail: I.cavanna@ausl.pc.it

ORCID: 0000-0002-0303-3778

Doi: $10.48286 / a r o .2021 .17$

\section{History}

Received: Apr 20, 2021

Accepted: May 20, 2021

Published: Jun 1, 2021

\section{ABSTRACT}

The published studies of patients with COVID-19 and cancer are done on hospitalized patients, and data on early outpatient treatment of symptomatic COVID-19 cancer patients are unavailable in English literature. This study included a retrospective, single-center case series of 20 consecutive symptomatic non-hospitalized adults with laboratory confirmed or probable COVID-19 infection. The diagnosis of pneumonia was done with portable ultrasound (US). The treatment was based on hydroxychloroquine (HCQ) plus azithromycin (AZ), drugs allowed during the first wave. Oxygen was delivered when necessary, and enoxaparine was done in bedridden patients. The primary endpoint was clinical improvement or hospitalization considered as worsening of clinical conditions. The secondary endpoints were
\end{abstract}

mortality at day 30 and at day 60 . A finger oximeter was delivered to each patient and all patients were monitored by tele-medicine, the side effects of treatment were registered and reported. From March 13rd to May 26th, 2020, 180 patients with laboratory - confirmed COVID-19 infection or with epidemiologically linked exposure to a person with laboratory - confirmed infection were managed at home for COVID-19 in the district of Piacenza, Emilia Romagna Region. Among these 180 patients, $20(11.11 \%)$ had cancer and form the basis of this report. There were 13 females (65\%) and 7 males $(35 \%)$, the majority of cancers were breast $(40 \%)$ and gastrointestinal (30\%). Seven patients (35\%) were on active anticancer treatment when infected and all with metastatic disease, 13 patients (65\%) 
were off therapy. Only one patient was hospitalized (5\%) and no patients died at 30 and 60 days. All 20 patients overcame the infection. The early home management of COVID-19 cancer patients allowed

\section{KEY WORDS}

SARS-COV-2; cancer; outpatients; COVID-19;

telemedicine; hospitalization rate. a very low hospitalization rate and no deaths were registered in this series. We believe that COVID-19 cancer patients should be treated precociously at home and monitored by telemedicine.

\section{IMPACT STATEMENT}

Although a small patient cohort was analyzed, our experience showed that the early diagnosis and treatment of COVID-19 infection in cancer patients could avoid hospitalization and death, prompting the implementation of early home management and monitoring through telemedicine.

\section{INTRODUCTION}

A novel coronavirus named coronavirus disease 2019 (COVID-19) emerged in Wuhan, China, in December 2019, and it has quickly spread globally (1). Cancer patients might be at higher risk of acquiring infection because poor general conditions, systemic immunosuppressive state caused by cancer itself and/or anticancer treatment such as chemotherapy, radiation, surgery, steroids, etc. In addition cancer patients have frequent scheduled visits to hospitals and clinics that can increase the risk to catch COVID-19 (2). The district of Piacenza, Emilia Romagna Region, is very near to the epicenter of the outbreak of COVID-19 in Italy (10 minutes by car), where on February $21^{\text {st }}$ there was the first Italian case, and the catastrophic nature of North Italy outbreak has been widely publicized $(3,4)$. The National Health Commission published guidelines that classified SARS-CoV-2 infections in 4 groups (mild type, moderate type, severe type and critical type) $(5,6)$. During the first pandemic wave, hydroxychloroquine (HCQ) with or without antiviral treatment has been initially incorporated in our national guidelines to treat $\operatorname{COVID}-19(7,8)$, and it was available until May $26^{\text {th }}, 2020$. In the district of Piacenza, a week later the $21^{\text {st }}$ February 2020, the Emergency Department of the Piacenza Hospital was overcrowded with COVID-19 infected people already in serious conditions. All these patients had a story of days or weeks spent at their home with fever, cough, fatigue, exertional dyspnea at first, then dyspnea at rest, that finally required the urgent hospitalization. All these patients were treated at home only with antipyretics like paracetamol, several with large spectrum antibiotics, like amoxicillin, and not any with HCQ or with antiretroviral or azithromycin (AZ). Since the treat-

ment of patients who were admitted to the Piacenza hospital, was based on HCQ tablets $400 \mathrm{mg}$ daily plus darunavir and cobicistat (DC) 800/150 daily for 7 days (8), we decided to perform strategic interventions to treat early at home COVID-19 infected cancer patients at the onset of symptoms avoiding to rest at home without medical care and avoiding the rapid progressive worsening of the patients. In this study, we did a comprehensive retrospective evaluation of cancer patients with COVID-19 infection, mild, moderate and severe type, precociously treated at home, in the district of Piacenza and neighboring areas from March 13 to May 26 $6^{\text {th }}, 2020$.

\section{MATERIALS AND METHODS}

At the end of February 2020, the district of Piacenza (North Italy), a taskforce program finalized to diagnosis, treatment, check and monitoring of patients affected by COVID-19, was constituted with units, formed by a medical doctor and a nurse equipped with personal protective equipment (PPE), medical car, portable ultrasound (US), portable electrocardiography finger oximeters, kits for nasopharyngeal swab and for blood samples, kits of drugs with: DC 800/150 mg, HCQ tablets 200 mg, steroids, prednisone $25 \mathrm{mg}$, enoxaparin subcutaneously 4.000 or 6.000 Unit and AZ tablets 500 mg. Patients with suspected symptoms of COVID-19 infection such as fever, cough, dyspnea can directly call the task force or call their family doctor, that activate the task force and the patients are promptly visited at their home. This program was called "The Piacenza Model". The COVID-19 severity was classified as 
follows in accordance with the diagnosis and treatment of COVID-19 guidelines (5): mild type: the clinical symptoms are mild with no abnormal radiological findings; moderate type: fever, cough and other symptoms are presented with pneumonia on chest computed tomography or sonography; severe type: disease is classified as if one of the following conditions is met: respiratory distress, respiratory rate $\geq$ 30 per men; oxygen saturation on room air at rest $\leq 93 \%$; partial pressure of oxygen in arterial blood / fraction of inspired oxygen $\leq 300 \mathrm{mmHg}$. Critical Type: one of the following conditions has to be met: respiratory failure occurs and mechanical ventilation is required; shock occurs; patients with other organ dysfunction need intensive care unit monitoring treatment. Chest ultrasound is performed as previously reported by an expert physician $(9,10)$ and nasopharyngeal swab specimens are collected according to the Center for Disease Central and Prevention Guidelines and performed with reverse transcription polymerase chain reaction (RT-PCR) (11). For patients without a recent electrocardiogram (ECG) and whit history of heart disease, ECG was carried out to allow HCQ therapy. The diagnosis of COVID-19 was based on typical symptoms, lung involvement on chest ultrasonography, and confirmed by RT-PCR for SARS-COV-2 detection in the majority of patients. However, in patients without RT-PCR for SARS-COV-2 detection, the diagnosis of COVID-19 was based on compatible symptoms after a high risk exposure. Patients with mild type of COVID-19 infection and without risk factors were treated only with symptomatic drugs like paracetamol; treatment with HCQ was reserved to patients with mild type presenting at least one of these risk factors: age $\geq 60$ years, obesity, hypertension, diabetes, cancer, Chronic Obstructive Pulmonary Disease (COPD), chronic ischemic heart disease. For patients with COVID-19 pneumonia causing respiratory illness, the treatment is immediately started with HCQ 400 mg twice daily on day one followed by $200 \mathrm{mg}$ twice daily alone for six days or HCQ plus AZ 500/daily for 6 days. A finger oximeter was delivered to each patient and every day the value of oxygen saturation and clinic symptoms are communicated by the patient on a phone or tablet to the task force's nurse and registered three times/day. When oxygen saturation $\mathrm{SaO} 2$ is of 93 or less, oxygen therapy is delivered at home. For bedridden patients, enoxaparine 4.000 to $6.000 \mathrm{UI} /$ daily subcutaneously is done, and when body temperature is above 38 ${ }^{\circ} \mathrm{C}$, paracetamol $500 \mathrm{mg}$ is recommended. Pred- nisone $35.5 \mathrm{mg}$ daily was added to patients with severe dyspnea. Treatment was offered to male and no pregnant female patients, 18 years or older. The treatment with HCQ was not performed in patients with QTc prolonged or with a story of favism. In this study, we report data of oncologic patients with laboratory-confirmed COVID-19 infection.

\section{Patient monitoring}

Patients were monitored on a phone or tablet by doctors or trained nurses. Three times per day for the evaluation of these parameters: finger oximeter, fever, cough, dyspnea, fatigue, other symptoms, ability to eat, to take drugs. Adverse effects of drugs were monitored. Patients with moderate or severe COVID-19 type were revisited every three days until they recovered. Hospitalization was evaluated for patients with progressive dyspnea.

\section{Patient outcome measuring}

The primary endpoint of this program was clinical improvement or hospitalization. Secondary endpoint was mortality at day 30 and at day 60 . Discontinuation of oncologic treatment was registered; adverse events related to the treatment were classified according to the National Cancer Institute Common Terminology Criteria for Adverse Events, Version N. 5 and reported (12).

\section{Statistical analysis}

Patients were registered with a unique recognition code, for everyone we recorded in a Microsoft Excel file (Microsoft Office version 2010): age, sex, symptoms and date of onset, comorbidities, COVID-19 therapy, COVID-19 severity, oxygen therapy, hospitalization and outcome. Quantitative variables are described by mean \pm standard deviation; qualitative variables are described by absolute and percentage frequencies. Comparisons of covariates were conducted using chi2 or Fisher's exact test for categorical variables and t-test or Mann Whitney test for continuous variables. All analyses were performed using STATA version 16 statistical software, with 2-sided significance tests and the 5\% significance level. This retrospective study was approved by the Local Ethics Committee (Institutional review board IRB (approval number 494/2020/OSS*/AUSLPC) ASL of Piacenza. The informed consent was obtained from each patient. In this retrospective study, we analyzed the data of 20 consecutive cancer patients affected by SARSCOV-2 diagnosed and treated at home. 


\section{RESULTS}

From March 13 to May 26 ${ }^{\text {th }}, 2020180$ patients with laboratory-confirmed COVID-19 infection were visited and treated at home, 72 men and 108 women, the mean age was $58.10 \pm 15.63$ years (range 18-91), 97 (53.88\%) patients showed one or more comorbidities, $20(11.11 \%)$ had cancer. We report here the results of 20 consecutive cancer patients with COVID-19. Clinical and demographic characteristics are reported in table I. There were 13 females (65\%) and 7 males (35\%), the majority of cancers were breast (40\%) and gastrointestinal (30\%), the other patients had lung (5\%), prostate(5\%), endometrium (10\%) and neuroendocrine cancer (10\%). Seven patients (35\%), all with metastatic disease, were on active anticancer

\begin{tabular}{|c|c|c|c|c|c|}
\hline \multirow[b]{2}{*}{ CHARACTERISTICS } & \multirow{2}{*}{$\begin{array}{l}\text { PATIENTS } \\
(\text { TOTAL }=20)\end{array}$} & \multicolumn{3}{|c|}{ SEVERITY OF COVID-19 } & \multirow[b]{2}{*}{ P-VALUE } \\
\hline & & MILD $(\mathbf{N}=7)$ & $\begin{array}{l}\text { MODERATE } \\
(\mathbf{N}=10)\end{array}$ & $\begin{array}{l}\text { SEVERE } \\
(N=3)\end{array}$ & \\
\hline Age mean $\pm d s$, range & $\begin{array}{c}61.85 \pm 12.27 \\
(32-83)\end{array}$ & $\begin{array}{l}63.14 \pm 13.61 \\
(46-83)\end{array}$ & $\begin{array}{c}59.3 \pm 13.13 \\
(32-75)\end{array}$ & $\begin{array}{c}67.33 \pm 3.79 \\
(63-70)\end{array}$ & 0.70 \\
\hline \multicolumn{6}{|c|}{ GENDER } \\
\hline Male n (\%) & $7(35)$ & $4(57.14)$ & $8(80)$ & $2(66.67)$ & \multirow{2}{*}{0.34} \\
\hline Female n (\%) & $13(65)$ & $3(42.85)$ & $2(20)$ & $1(33.33)$ & \\
\hline \multicolumn{6}{|c|}{ COMORBIDITIES N (\%) } \\
\hline Hypertension & $4(20)$ & $1(14.29)$ & $2(20)$ & $1(33.33)$ & 1 \\
\hline $\begin{array}{l}\text { Chronic obstructive } \\
\text { pulmonary disease } \mathrm{n}(\%)\end{array}$ & $4(20)$ & $2(28.57)$ & $2(20)$ & $0(0)$ & 1 \\
\hline Diabetes n (\%) & $4(20)$ & $2(28.57)$ & $1(10)$ & $1(33.33)$ & 0.44 \\
\hline Coronary disease n (\%) & $0(0)$ & $0(0)$ & $0(0)$ & $0(0)$ & \\
\hline Other n (\%) & $4(20)$ & $2(28.57)$ & $1(10)$ & $1(33.33)$ & 0.44 \\
\hline \multicolumn{6}{|c|}{ TUMOR SITE } \\
\hline Breast & $8(40)$ & $2(28.57)$ & $5(50)$ & $1(33.33)$ & \multirow{3}{*}{0.36} \\
\hline Gastrointestinal & $6(30)$ & $1(14.29)$ & $3(30)$ & $2(66.67)$ & \\
\hline Other & $6(30)$ & $4(57.14)$ & $2(20)$ & $0(0)$ & \\
\hline \multicolumn{6}{|c|}{ ACTIVE CANCER TREATMENT } \\
\hline Yes & $7(35)$ & $3(42.86)$ & $4(40)$ & $0(0)$ & \multirow{2}{*}{0.57} \\
\hline No & $13(65)$ & $4(57.14)$ & $6(60)$ & $3(100)$ & \\
\hline \multicolumn{6}{|c|}{ SYMPTOMS } \\
\hline Fever n (\%) & $19(95)$ & $7(100)$ & $9(90)$ & $3(100)$ & 1 \\
\hline Cough n (\%) & $13(65)$ & $3(42.86)$ & $8(80)$ & $2(66.67)$ & 0.34 \\
\hline Dyspnea n (\%) & $5(25)$ & $0(0)$ & $3(30)$ & $2(66.67)$ & 0.09 \\
\hline Diarrhea n (\%) & $5(25)$ & $2(28.57)$ & $2(20)$ & $1(33.33)$ & 1 \\
\hline Fatigue n (\%) & $3(15)$ & $1(14.29)$ & $2(20)$ & $0(0)$ & 1 \\
\hline Dysgeusia n (\%) & $0(0)$ & $0(0)$ & $0(0)$ & $0(0)$ & \\
\hline Other n (\%) & $5(25)$ & $4(57.14)$ & $1(10)$ & $0(0)$ & 0.06 \\
\hline $\begin{array}{l}\text { Duration of symptoms } \\
\text { before treatment (days) } \\
\text { mean } \pm \text { ds, range }\end{array}$ & $\begin{array}{c}6.35 \pm 2.81 \\
(3-12)\end{array}$ & $\begin{array}{l}6.14 \pm 2.80 \\
(3-10)\end{array}$ & $\begin{array}{l}5.9 \pm 3.07 \\
(3-12)\end{array}$ & $\begin{array}{c}8.33 \pm 1.53 \\
(7-10)\end{array}$ & 0.34 \\
\hline $\begin{array}{l}\text { Treatment for COVID-19 } \\
(\mathrm{HCQ}+\mathrm{AZ})\end{array}$ & $19(95)$ & $6(85.71)$ & $10(100)$ & $3(100)$ & 0.5 \\
\hline \multicolumn{6}{|c|}{ OUTCOME } \\
\hline Dead at 30 days & $0(0)$ & $0(0)$ & $0(0)$ & $0(0)$ & \\
\hline Dead at 60 days & $0(0)$ & $0(0)$ & $0(0)$ & $0(0)$ & \\
\hline Hospitalized n (\%) & $1(5)$ & $0(0)$ & $0(0)$ & $1(33.33)$ & 0.15 \\
\hline
\end{tabular}

Table I. Clinical and demographiccharacteristics ofoncologic COVID-19 patients diagnosed and treated athome based on severity of COVID-19. AZ: azithromycin; HCQ: hydroxychloroquine. 
treatment ( 2 chemotherapy, 2 immunotherapy and 3 hormone therapy), while 13 patients (65\%) were not. The most frequent symptoms were fever (95\%) and cough (65\%). 7 (35\%) patients showed mild, 10 (50\%) moderate and 3 (15\%) severe COVID-19 type (5). The most frequent comorbidities were hypertension $(20 \%)$, diabetes (20\%) and chronic obstructive pulmonary disease (20\%). The duration of symptoms before treatment was higher in patients with severe COVID-19 (8.33 \pm 1.53 days) when compared to patients with moderate (5.9 \pm 3.07 days) and mild type (6.14 \pm 2.80 days). 19 patients were treated with lowdose HCQ (800 mg day 1, subsequently 400 mg daily for 6 additional days), plus AZ (500 mg daily for 6 days). 1 patient with mild COVID-19 type was treated with non-steroid anti-inflammatory drugs. Primary endpoint: only 1 patient (5\%), with stage III pancreatic cancer, not on active treatment, was hospitalized and was discharged after 3 days; secondary endpoint: no patients died at 30 and 60 days. The treatment with HCQ and AZ was well tolerated in the 19 cancer patients, only 1 patient (5.26\%) had diarrhea and $1(5.26 \%)$ headache and abdominal pain.

\section{DISCUSSION}

In this report, we describe the first series of cancer patients with COVID-19 treated early at home in a western country. Italy has been the first country in Europe experiencing an outbreak of COVID-19 and Piacenza is very near to the epicenter of Lombardy's outbreak. Oncology scientific societies have quickly released guidelines on cancer care during the pandemic, suggesting to use telemedicine to reduce the spread of infection (13-15). We are aware that our results provide some descriptive information, but the prognostic role of these findings is still potential and should be clarified by further and large-scale studies. These 20 cancer patients with COVID-19 infection manifested respiratory symptoms including fever, cough and shortness of breath. In our study, there were 3 severely ill patients, 10 moderate and 7 mild patients. However, we observed that the disease of most patients showed a moderate course. In particular, only 1 (5\%) of those 20 patients was hospitalized for progressive dyspnea and discharged after 3 days. We previously reported 25 cancer patients with COVID-19 treated in our hospital: nine (36\%) of these patients died, while 16 (64\%) overcame the infection (8). In a second report (16), a retrospective study was performed in the hospitals of our district, including 51 hospitalized cancer patients with COVID-19, 25 of these 51 patients (49\%) died, 12 of 51 (23.53\%) owing to cancer and 13 of 51 (25.49\%) owing to COVID-19. In our opinion, with the limits of retrospective studies and with different settings of cancer patients with COVID-19 (likely more serious and advanced disease in hospitalized patients), the early COVID-19 treatment allowed a more favorable outcome. It must be emphasized that COVID-19 hospitalized patients, above all, when critically ill, may be associated with hospital-acquired infections (HAls). A recent study showed that $46 \%$ of 774 COVID-19 hospitalized patients developed 759 HAIDs and 234 patients (30\%) died in ICU (17). These data support the strategy to treat early at home (when possible) cancer patients with COVID-19 avoiding hospitalization and death. The integration between early treatment and home management of COVID-19 patients could be an element of success in the management of the emergency. However, further assessments are required on the effectiveness and impact of the adopted model.

\section{CONCLUSIONS}

Although a small patient cohort was analyzed, our experience showed that the early diagnosis and treatment of COVID-19 infection in cancer patients could avoid hospitalization and death, prompting the implementation of early home management and monitoring through telemedicine.

\section{ETHICS}

\section{Fundings}

There were no institutional or private fundings for this article.

\section{Conflict of interests}

The authors have declared no conflict of interests.

\section{Availability of data and material}

The data underlying this article can be shared just before a reasonable request to the corresponding author.

\section{Authors' contribution}

All the authors contributed equally to conception, data collection, analysis and writing of this paper. 


\section{REFERENCES}

1. World Health Organization. Coronavirus disease (COVID-19) outbreak 2020. Available at: https://www.who.int/director-general/speeches/detail/who-director-general-s-opening-remarks-at-the-media-briefing-on-covid-19---11march-2020.

2. Izcovich A, Ragusa MA, Tortosa F, et al. Prognostic factors for severity and mortality in patients infected with COVID- 19: A systematic review. PLoS One 2020;15(11):e0241955. Doi: 10.1371/journal.pone.0241955.

3. Worldometer. COVID-19 coronavirus pandemic 2020. Available at: https://www.worldometers. info/coronavirus/. Last access: Apr 162021.

4. Horowitz J. Italy's healthcare system groans under coronavirus-a warning to the world. New York Times. Available at: https://www. nytimes.com/2020/03/12/world/europe/12italy-coronavirus-health-care.html ). Last access: March 122020.

5. Feng Y, Ling Y, Bai T, et al. COVID-19 with Different Severities:AMulticenter Study of Clinical Features. Am J Respir Crit Care Med 2020;201(11):1380-8. Doi: 10.1164/rccm.202002-04450C.

6. Lai CC, Liu YH, Wang CY, et al. Asymptomatic carrier state, acute respiratory disease, and pneumonia due to severe acute respiratory syndrome coronavirus 2 (SARSCoV-2): Facts and myths. J Microbiol Immunol Infect 2020;53(3):404-12. Doi: 10.1016/j. jmii.2020.02.012.

7. Linee guida SIMIT: pazienti con infezione da coronavirus COVID-19. Available at: https://www.npsitalia.net/wp-content/uploads/2020/03/protocolloterapeutico.pdf. Last access: 3 Mar 2020.

8. Stroppa EM, Toscani I, Citterio C, et al. Coronavirus disease-2019 in cancer patients. A report of the first 25 cancer patients in a western country (Italy). Future Oncol 2020;16(20):142532. Doi: 10.2217/fon-2020-0369.

9. Nouvenne A, Zani MD, Milanese G, et al. Lung Ultrasound in COVID-19 Pneumonia:
Correlations with Chest CT on Hospital admission. Respiration 2020;99(7):617-24. Doi: 10.1159/000509223.

10. Yang $W$, Sirajuddin A, Zhang $X$, et al. The role of imaging in 2019 novel coronavirus pneumonia (COVID-19). Eur Radiol 2020;30(9):4874-82. Doi: 10.1007/s00330-020-06827-4.

11. Centers for Disease Control and Prevention. Interim guidelines for collecting, handling, and testing clinical specimens from patients under investigation (PUIs) for 2019 novel coronavirus (2019-nCoV). Available at: https://www.cdc.gov/ coronavirus/2019-ncov/lab/guidelines-clinicalspecimens.html. Last access: 16 Apr 2021.

12. Available at: https://ctep.cancer.gov/protocoldevelopment/electronic_applications/docs/ ctcae_v5_quick_reference_8.5x11.pdf

13. Darcourt JG, Aparicio K, Dorsey PM, et al. Analysis of the Implementation of Telehealth Visits for Care of Patients With Cancer in Houston During the COVID-19 Pandemic. JCO Oncol Practice 2021;17:1,e36-e43.

14. Indini A, Aschele C, Cavanna L, et al. Reorganisation of medical oncology departments during the novel coronavirus disease-19 pandemic: a nationwide Italian survey. Eur J Cancer 2020;132:17-23. Doi: 10.1016/j. ejca.2020.03.024.

15. Brandes AA, Ardizzoni A, Artioli F. et al. Fighting cancer in coronavirus disease era: organization of work in medical oncology departments in Emilia Romagna region of Italy. Future Oncol 2020;16(20):1433-39. Doi: 10.2217/fon2020-0358.

16. Cavanna L, Citterio C, Toscani I, et al. Cancer patients with COVID-19. A retrospective study of 51 patients in the district of Piacenza, Northern Italy. Future Sci OA 2020;7(1):FSO645. Doi: 10.2144/fsoa-2020-0157.

17. Grasselli G, Scaravilli V, Mangioni D, et al. Hospital-acquired infections in critically-ill COVID-19 patients. Chest. 2021;S0012-3692(21)00679-6. Doi: 10.1016/j.chest.2021.04.002. 